\title{
GRADIENT CORRELATION METHOD FOR THE STABILIZATION OF INVERSION RESULTS OF AEROSOL MICROPHYSICAL PROPERTIES RETRIEVED FROM PROFILES OF OPTICAL DATA
}

\author{
Alexei Kolgotin ${ }^{1}$, Detlef Müller, ${ }^{2,3}$ Anton Romanov ${ }^{4}$, Eduard Chemyakin ${ }^{2}$ \\ ${ }^{(1)}$ Physics Instrumentation Center, Troitsk, Moscow Region, 142190, Russia, alexeift@yahoo.com \\ ${ }^{(2)}$ Science Systems and Applications, Inc. (SSAI), NASA LaRC, \\ 1 Enterprise Parkway, Hampton, VA, 23666, USA, \\ ${ }^{(3)}$ University of Hertfordshire, Hatfield, Hertfordshire, AL10 9AB, UK, d.mueller@herts.ac.uk \\ ${ }^{(4)}$ The National University of Science and Technology, Leninskii av. 4, Moscow, 119049
}

\begin{abstract}
Correlation relationships between aerosol microphysical parameters and optical data are investigated. The results show that surface-area concentrations and extinction coefficients are linearly correlated with a correlation coefficient above 0.99 for arbitrary particle size distribution. The correlation relationships that we obtained can be used as constraints in our inversion of optical lidar data. Simulation studies demonstrate a significant stabilization of aerosol microphysical data products if we apply the gradient correlation method in our traditional regularization technique.
\end{abstract}

\section{INTRODUCTION}

Several approaches for the retrieval of particle microphysical parameters from multiwavelength lidar measurements were developed in the past years [1-3]. Since we deal with an ill-posed, illconditioned problem the derived solutions oscillate, they are unstable, and we may obtain nonphysical results. Even the use of advanced mathematical methods such as regularization, e.g. Tikhonov's regularization does not guarantee that we find physically meaningful solutions unless we introduce additional constraints in the solution spaces. We showed that we can stabilize the solution space if we use whole sections of optical data profiles rather than optical data sets of individual height bins as input in our inversion scheme [4].

If the profiles of the optical data (OD) do not change, in other words the profile gradient is close to 0 , we can expect that profiles of particle microphysical parameters (PMP) do not vary significantly either. On the contrary if the OD profiles show variations, i.e., the "gradient" profile differs from 0, we can expect that the PMP vary with height, too. In this contribution we investigated which correlations between lidar optical data and particle microphysical parameters exist and if these correlations can be used in our inver- sion methodology for improving the microphysical data products.

In section 2 we present the correlation relationships we found between PMP and OD. We describe how they can be used in our gradient correlation method. In section 3 we show a numerical example with synthetic OD. Retrieval results of different approaches are compared in section 4. Section 5 summarizes our results.

\section{METHODOLOGY}

Lidar measurements deliver the OD which can be used for the retrieval of bulk PMP $(p)$ such as mean radius $\left(r_{\text {mean }}\right)$ and effective radius $\left(r_{\text {eff }}\right)$, mean width, i.e. geometrical standard deviation $(\sigma)$ of a particle size distribution, number $(n)$, surface-area $(s)$ and volume $(v)$ concentrations. This problem is related to solving the Fredholm integral equation of the $1^{\text {st }}$ kind

$$
\begin{gathered}
\int_{r_{\min }^{(l)}}^{r_{\max }} K_{g}\left(\lambda, m^{(l)}, r\right) f^{(l)}(r) d r=g^{(l)}(\lambda), \\
l=1, . ., \mathrm{N}_{\mathrm{L}}, g=\alpha, \beta
\end{gathered}
$$

The optical data $g^{(l)}(\lambda)$ are measured with lidar at wavelength $\lambda$. The unknown function $f^{(l)}(r)$ describes the particle size distribution (PSD), and $r$ describe the particle radius on the domain $\left[r_{\min }{ }^{(l)}, r_{\max }{ }^{(l)}\right]$. Here we consider $r_{\max }{ }^{(l)}>0.05 \mu \mathrm{m}$. The kernel functions $K_{g}(\lambda, m, r)$ can be computed on the basis of Mie-scattering theory in the case of spherical particle shape. The parameter $m^{(l)}=m_{R}^{(l)}-i m_{I}^{(l)}$ describes the particle complex refractive index. Extinction coefficients are denoted as $g=\alpha$, backscatter coefficients are denoted as $g=\beta$. The superscript $l$ indicates the number of the height bin we use to describe the OD profiles. The total number of height bins is $\mathrm{N}_{\mathrm{L}}$. The PMP can be easily estimated from the solution $f$.

Eq. (1) can be solved, for example, with regularization which allows us to find a solution space $F^{(l)}=\left\{f_{1}^{(l)}(r), \ldots, f_{\mathrm{Nsol}}^{(l)}(r)\right\}$ at each height bin $l$. If we know $F^{(l)}$ we can also obtain the solution 
spaces for any bulk parameter $P^{(l)}=\left\{p_{1}{ }^{(l}, \ldots\right.$, $\left.p_{\text {Nsol }}^{(l)}\right\}$ at each height bin $l$, where $P^{(l)}=R_{\text {mean }}{ }^{(l)}$, $R_{\mathrm{eff}}^{(l)}, \Sigma^{(l)}, N^{(l)}, S^{(l)}$, or $V^{(l)}$ denotes the solution space of $r_{\text {mean }}, r_{\text {eff }}, \sigma, n, s$, or $v$ respectively [1]. The collection of the solution spaces for all $\mathrm{N}_{\mathrm{L}}$ heights forms the profiles of the PMP. The final solution $p_{\mathrm{av}}$ at height $l$ is defined by averaging all individual parameters $p_{i}^{(l)}, i \leq \mathrm{N}_{\text {sol }}$, over some interval of the discrepancy range $\left[\rho_{\min }{ }^{(l)}, \rho_{\mathrm{av}}{ }^{(l)}\right][2,4]$. In order to find physically meaningful solutions we need to apply different constraints, as for example, the discrepancy range $\left[\rho_{\min }{ }^{(l)}, \rho_{\mathrm{av}}{ }^{(l)}\right]$, the radius range $\left[r_{\min }{ }^{(l)}, r_{\max }{ }^{(l)}\right]$, and the complex refractive index $m^{(l)}$. In that way we can exclude individual solutions $p_{i}^{(l)}$ which for example deviate too much from the average value $p_{\text {av }}$. In that case we insert a threshold $\delta p$ to take into consideration only those individual solutions that fulfill the condition

$$
\left|p_{i}^{(l)}-p_{\text {av }}\right|<\delta p
$$

In our previous strategy of data inversion we used this averaging procedure for each solution space (in each height bin) independently, i.e., we did not take into consideration that results of successive height bins may be correlated to each other. The profile of the OD contains information regarding the variation of the profile of the PMP. In other words we can predict the PMP behavior if we know the law(s) (or correlations) that describes the interdependence of OD and PMP.

The interdependence that is generally accepted is that effective radius $r_{\text {eff }}$ is inversely proportional to the extinction Ångström exponent (EAE) $\gamma$ :

$$
\gamma=\ln \left[\alpha\left(\lambda_{1}\right) / \alpha\left(\lambda_{2}\right)\right] / \ln \left(\lambda_{2} / \lambda_{1}\right) \text {. }
$$

For small variations $\delta \gamma$ we obtain

$$
r_{\text {eff }}=a_{r} \gamma+b_{r} \text {. }
$$

The constants $a_{r}$ and $b_{r}$ are regression coefficients (RC). The linear correlation (4) can be used as extra constraint if we want to find the solution space for $R_{\mathrm{eff}}^{(l)}$. In fact if we measure $\gamma^{(l)}$ the correlation (4) allows us to estimate $r_{\mathrm{eff}}^{(l)^{*}}$ according to

$$
r_{\text {eff }}^{(l)^{*}}=a_{r} \gamma^{(l)}+b_{r}
$$

If we introduce the threshold $\delta r_{\text {eff }}$ we can rewrite condition (2) as

$$
\left|r_{\mathrm{eff}, i}^{(l)}-r_{\mathrm{eff}}^{(l)^{*}}\right|<\delta r_{\mathrm{eff}}
$$

If we approximate the kernel $K_{\alpha}$ and the PSD $f$ by the parabolic and rectangle functions, respectively, one can show on the basis of the average theorem that there are additional linear correlations between extinction (in $\mathrm{Mm}^{-1}$ ) and other PMP and combinations of several PMP (in $\mathrm{Mm}^{-1}$ ) with correlation coefficients $R^{2}>0.99$ :

$$
s=a_{s} \alpha(\lambda)+b_{s},
$$

$$
\begin{gathered}
v / r_{\text {eff }}=a_{v} \alpha(\lambda)+b_{v}=\left[a_{s} \alpha(\lambda)+b_{s}\right] / 3, \\
n r_{\text {eff }}^{2}=a_{n} \alpha(\lambda)+b_{n}
\end{gathered}
$$

The correlation (9) is valid only for a fixed standard deviation $\sigma$.

The linear correlations (7)-(9) and relationship (4) can be used as extra constraints to identify the solution spaces. We stress that any combination of correlations (4) and (8), (9), is acceptable, for example

$$
v /\left(a_{r} \gamma+b_{r}\right) \approx\left[a_{s} \alpha(\lambda)+b_{s}\right] / 3 .
$$

Again, introducing the threshold $\delta p$ the condition (6) and the correlations (4), (8), (9) can be rewritten in a more general form as

$$
\left|p_{i}^{(l)}-p^{(l)^{*}}\right|<\delta p \quad p=r_{\mathrm{eff}}, s, v / r_{\mathrm{eff}}, n r_{\mathrm{eff}}^{2}(11)
$$

and

$$
p^{(l)^{*}}=a_{p} x^{(l)}+b_{p} \quad x=\alpha, \gamma
$$

The use of the constraints (11) in the averaging procedure $[2,4]$ is at the heart of the gradient correlation method (GCM).

\section{NUMERICAL SIMULATION \\ 3.1. Synthetic optical data}

We used synthetic optical data (SOD) to identify the correlation relationships. We generated the synthetic optical data $3 \beta+2 \alpha$ at 355,532 and $1064 \mathrm{~nm}$ for backscatter coefficients and at 355 and $532 \mathrm{~nm}$ for extinction coefficients [1]. We took into account Eq. (1). We pre-defined values for $f$ and $m$. We used the lognormal law for describing $f$. We selected a wide range of values for the mean radius, i.e. $r_{\text {mean }}=20,60,100,140,180$, 220,260 , and $300 \mathrm{~nm}$. With regard to the mode width we used $\sigma=1.5,1.7,1.9,2.1,2.3$, and 2.5. We selected the following values for the real and imaginary parts: $m_{R}=1.4,1.5,1.6,1.7$, and $m_{I}=0$, $1 \mathrm{e}-4,1 \mathrm{e}-3,2.5 \mathrm{e}-3,5 \mathrm{e}-3,7.5 \mathrm{e}-3,1 \mathrm{e}-2,1.5 \mathrm{e}-2,2 \mathrm{e}-$ $2,2.5 \mathrm{e}-2,3 \mathrm{e}-2,3.5 \mathrm{e}-2,4 \mathrm{e}-2,4.5 \mathrm{e}-2$, and $5 \mathrm{e}-2$. We used all possible combinations $\left(r_{\text {mean }}, \sigma, m_{R}\right.$, $\left.m_{I}\right)$ and in that way we created 2880 sets of $3 \beta+2 \alpha$ data for the SOD bank.

Fig. 1 presents the statistical analysis of the SOD bank. We see that $r_{\text {eff }}$ is nearly inversely proportional to $\gamma$ (Fig. 1a). The range of EAE varies from -0.5 to 4 . For example, if $\gamma>2$, which means that $\delta r_{\text {eff }}<0.1 \mu \mathrm{m}$, we find for the RC $a_{r}=-0.02$ and $b_{r}=0.1$. The analysis of all entries of the SOD bank shows that these two parameters vary in the diapasons:

$$
a_{r} \in[-0.02 ;-1.7], \quad b_{r} \in[0.1 ; 1.5]
$$

We find linear correlations of $R^{2}=0.998$ for $s$ versus $\alpha(355)$ [see Fig. 1b]. In the following we denote IP as parameters we investigated with regard to useful correlation properties. If we use all values stored in the SOD bank we find for the RC: 
$a_{s}=1.7$ and $b_{s}=-0.08$. A more thorough analysis of the SOD bank shows the following variations

$$
a_{s} \in[1.33 ; 1.75], \quad b_{s} \in[-0.05 ; 0.03](14)
$$

These values depend on $\sigma$. The strong correlation guarantees that surface-area concentration can be directly estimated from the extinction with an uncertainty that is less than the measurement error.

Fig. 1b shows that $v / r_{\text {eff }}$ and $\alpha$ are linearly correlated as well. It can be shown that Eq. (7) and (8) are true for any amount of modes in PSDs.

We also find a linear correlation of $R^{2}=0.999$ for IP $n r_{\text {eff }}^{2}$, but in that case $\sigma$ needs to be kept constant (see Fig. 1c). Depending on $\sigma \in[1.5 ; 2.5]$ we find the following RCs:
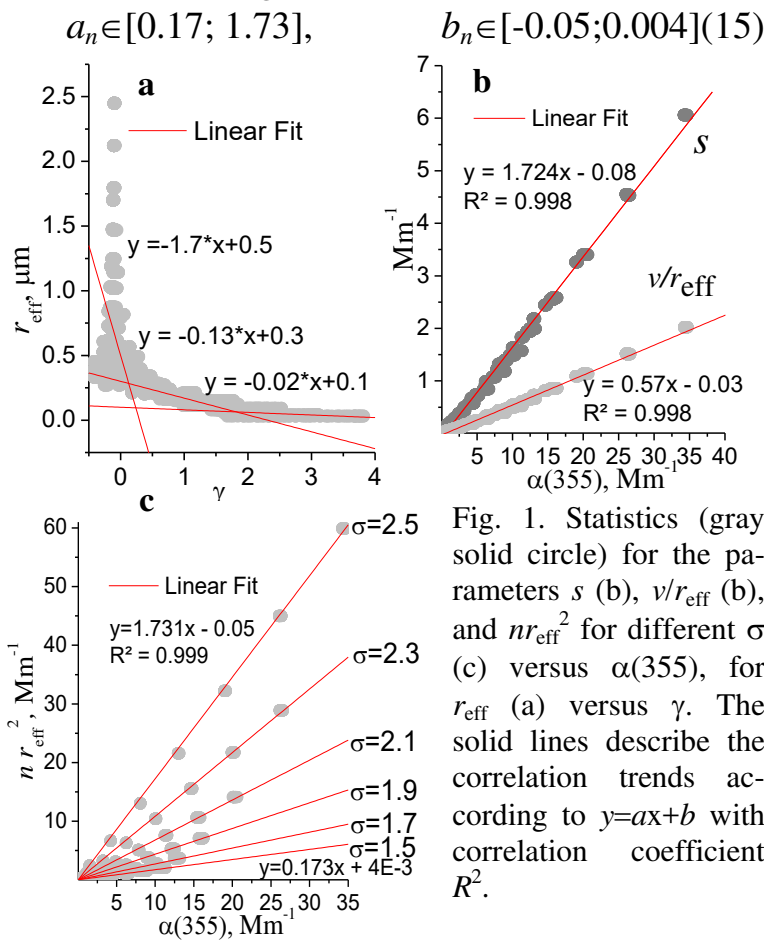

Fig. 1. Statistics (gray solid circle) for the parameters $s$ (b), $v / r_{\text {eff }}(\mathrm{b})$, and $n r_{\text {eff }}^{2}$ for different $\sigma$ (c) versus $\alpha(355)$, for $r_{\text {eff }}$ (a) versus $\gamma$. The solid lines describe the correlation trends according to $y=a \mathrm{x}+b$ with correlation coefficient $R^{2}$.

\subsection{Retrieval example for synthetic data}

We selected $\mathrm{N}_{\mathrm{L}}=6$ OD sets of $3 \beta+2 \alpha$ data from the SOD bank and we constructed OD profiles (see Fig. 2a). The true PMP are shown in Fig. 2b and $2 \mathrm{c}$ as thick lines. The real part does not change with height. It is equal to 1.5 . The imaginary part increases with height, i.e. $m_{l}=0,0.01$, 0.03 , and 0.05 (not shown). In the inversion problem the true OD profile are distorted with $15 \%$ extreme error.

In the next step we identified the solution space $F^{(l)}, l=1, \ldots, 6$, with our traditional regularization technique [2]. Each solution space $F^{(l)}$ contains about $6 \times 10^{4}$ individual solutions which are retrieved for 100 inversion windows that slip inside the radius range from 0.03 to $8 \mu \mathrm{m}$. We used 20 equidistant values of the real part, i.e.,
$m_{R} \in[1.325 ; 1.8]$ and 30 equidistant values of the imaginary part, i.e., $m_{I} \in[0.0 ; 0.1]$.

Finally we post-processed the solution spaces with GCM when the RCs $a_{p}$ are known (True $\mathrm{RC})$ and when they are distorted up to $25 \%$ (Dist.RC). In that way we could test the stability of our novel approach. In addition we postprocessed the solution spaces in the automated mode [5] without using GCM and without using any constraints of the parameters $r_{\text {eff }}, n$, $r \in[0.03 ; 8], \quad m_{R} \in[1.325 ; 1.8]$ and $m_{I} \in[0.0 ; 0.1]$ (NoGCM).

All retrieval results are shown in Fig. $2 b$ and Fig. 2c. We see that in the case of NoGCM (triangle) there are large retrieval errors of effective radius in 3 of the 6 height bins, and number concentration shows errors up to $200 \%$ in the height bin $l \# 4$. The solution can be significantly improved if we require that the correlation relationships (4), (9), and (10) are fulfilled. In the case of True CC (square) and Dist.CC (star) the retrieval errors of number concentration do not exceed $50 \%$, the error of effective radius is even less.

In order to obtain the GCM results we used the RCs shown in the table:

\begin{tabular}{|c|c|c|c|c|c|c|}
\hline \multirow{2}{*}{$p$} & $a_{p}$ & $b_{p}$ & $\delta p$ & $a_{p}$ & $b_{p}$ & $\delta p$ \\
\cline { 2 - 7 } & \multicolumn{3}{|c|}{ True RC } & \multicolumn{3}{c|}{ Dist.RC } \\
\hline$r_{\text {eff }}$ & -1.0 & 0.76 & 0.25 & -0.8 & 0.8 & 0.25 \\
\hline$\nu /\left(a_{r} \gamma+b_{r}\right)$ & 0.7 & -0.05 & 0.1 & 0.56 & -0.05 & 0.1 \\
\hline$n r_{\text {eff }}{ }^{2}$ & 0.7 & -0.04 & 0.1 & 0.5 & -0.04 & 0.1 \\
\hline
\end{tabular}

We present the statistics for all results derived with NoGCM (triangle), TrueRC (square) and DistRC (star) in Fig. 3. The statistics for the true data obtained from the SOD bank are plotted as circles in Fig. 3. Fig. 3 also shows the (actual) RCs, which we obtained after post-processing the solution space $F^{(l)}$, for all three cases together with RCs for the true data.

We see that the parameter $s$ that is retrieved with NoGCM, TrueRC, and DistRC is linearly correlated with extinction (Fig. 3b). We obtain $R^{2}=1$ and $a_{s}=1.7$ which is equal to the true value. This is the reason why we do not use Eq. (7) in GCM (see table).

We find outliers in the profiles of $r_{\text {eff }}$ and $n$ if we use NoGCM (see Fig. 2). As a result the actual RCs $a_{r}=1.82$ (Fig. 3a, triangle) and $a_{n}=2.37$ (Fig. $3 c$, triangle) are out of their ranges (13) and (15), respectively.

If we implement in GCM the relationships (4) and (9) and we use the RCs from the table, we find for the actual RCs: $a_{r}=1.14\left(R^{2}=0.95\right)$ and $a_{n}=0.68 \quad\left(R^{2}=0.999\right)$ for TrueRC (Fig. 3a, 3c, square). If we use GCM with DistRC we obtain 

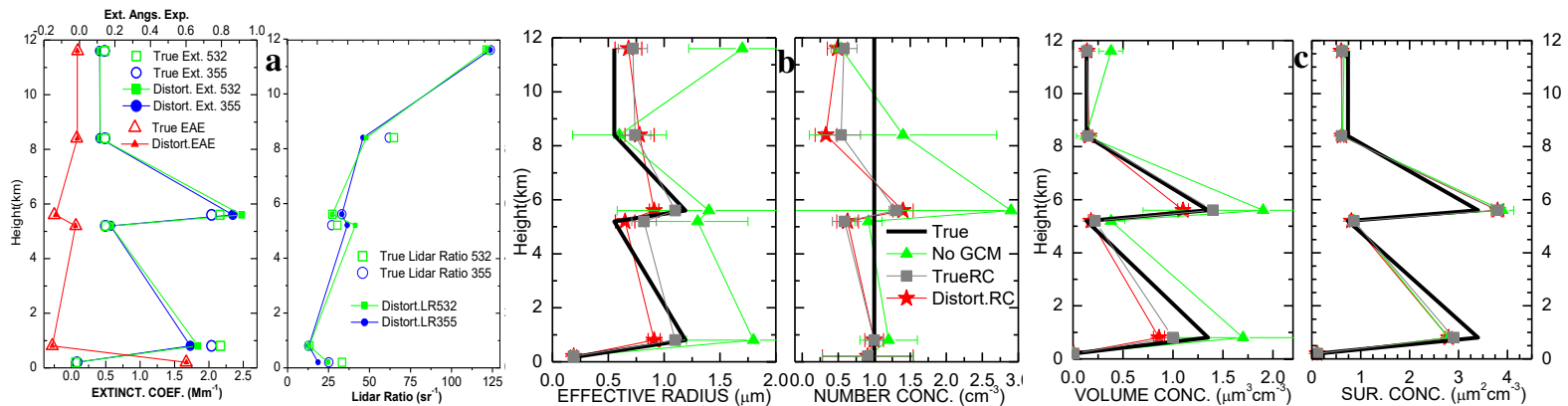

Fig. 2. Retrieval results for the case of a $3 \beta+2 \alpha$ data set constructed from our SOD. a: input true (open marker) and distorted (close marker+line) EAE, extinctions, lidar ratios at 355 and $532 \mathrm{~nm}$. b, c: true (thick line) and retrieved (line+marker) PMP. Retrieval results were obtained when GCM is not used (triangle), GCM is used with true RC (square), GCM is used with RC distorted up to $25 \%$.

RCs $a_{r}=0.93\left(R^{2}=0.97\right)$, and $a_{n}=0.49\left(R^{2}=0.999\right)$ [see Fig. 3a, 3c, star]. Notably the actual and true RCs converge.

\section{CONCLUSION}

We presented the novel method of GCM for the stabilization of PMP retrieved from profiles of optical data taken with lidar. GCM uses correlation relationships between particle bulk parameters and measured optical information.

We found that the linear correlation between particle surface-area concentration and extinction coefficient is approximately $R^{2} \approx 1$. The IP $v / r_{\text {eff }}$ and extinction coefficient are linearly correlated with the same correlation coefficient. We find a linear correlation between the product $n r_{\text {eff }}^{2}$ and particle extinction. The correlation is $R^{2} \approx 1$ for fixed mode width $\sigma$.

These relationships allow us to use additional constraints during the post-processing of the solution spaces. As a result the surface-area concentration can be estimated with an uncertainty that is less than the measurement error of the extinction coefficient.

Our comparisons between the results obtained with GCM and with our traditional regularization technique show a significant stabilization of the retrieved profiles of aerosol microphysical properties. We will continue with numerical simulations and the analysis of case studies in order to further assess the potential of this new approach.

\section{REFERENCES}

1. Müller D., et al., 1999: Microphysical particle parameters from extinction and backscatter lidar data by inversion with regularization: theory, Appl. Opt., 38, pp. 2346 - 2357.

2. Veselovskii I., et al., 2002: Inversion with regularization for the retrieval of tropospheric aerosol parameters from multiwavelength lidar sounding, Appl. Opt., 41, pp. 3685 - 3699.
3. Böckmann C., et al, 2005: Microphysical aerosol parameters from multiwavelength lidar, Journal of Optical Society of America, A, 22, pp. 518-528.

4. A. Kolgotin and D. Müller, 2008: Theory of inversion with two-dimensional regularization: profiles of microphysical particle properties derived from multiwavelength lidar measurements, Appl. Opt., 47, pp. 4472-4490.

5. D. Müller, et al, 2014: Airborne Multiwavelength High Spectral Resolution Lidar (HSRL-2) observations during TCAP 2012: vertical profiles of optical and microphysical properties of a smoke/urban haze plume over the northeastern coast of the US, Atmos. Meas. Tech. 7, 34873496, 2014.
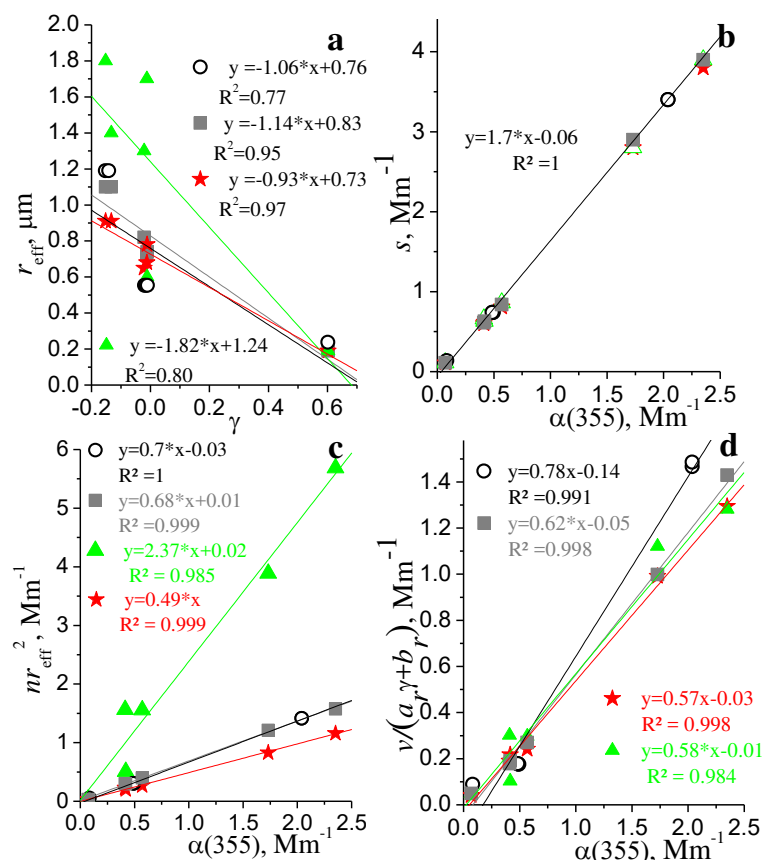

Fig. 3. Statistics as shown in Fig. 1 for true (circle), and retrieved values obtained from the use of the NoGCM (triangle), TrueRC (square) and Dist.RC (star) parameters: $s$ (b), $v / r_{\text {eff }}$ (d), and $n r_{\text {eff }}^{2}$ for different $\sigma$ (c) versus $\alpha(355)$, for $r_{\text {eff }}($ a) versus $\gamma$. The solid lines describe correlation trends according to $y=a \mathrm{x}+b$ and $R^{2}$. 